\title{
Controlled generation of atomic vacancies in chemical vapor deposited graphene by microwave oxygen plasma
}

\author{
R. Rozada ${ }^{\text {a* }}$, P. Solís-Fernández ${ }^{\text {, J.I. Paredes }}{ }^{\mathrm{a}}$, A. Martínez-Alonso ${ }^{\mathrm{a}}$, H. Ago ${ }^{\mathrm{b}}$, J.M.D. \\ Tascón $^{\mathrm{a}}$ \\ anstituto Nacional del Carbón, INCAR-CSIC, Apartado 73, 33080 Oviedo, Spain \\ ${ }^{b}$ Institute for Materials Chemistry and Engineering, Kyushu University, Fukuoka 816- \\ 8580, Japan
}

The introduction of atomic-scale defects in a controllable manner and the understanding of their effect on the characteristics of graphene are essential to develop many applications based on this two-dimensional material. Here, we investigate the use of microwave-induced oxygen plasma towards the generation of small-sized atomic vacancies (holes) in graphene grown by chemical vapor deposition. Scanning tunneling microscopy revealed that tunable vacancy densities in the $10^{3}-10^{5} \mu \mathrm{m}^{-2}$ range could be attained with proper plasma parameters. Transport measurements and Raman spectroscopy revealed p-type doping and a decrease in charge carrier mobility for the vacancy-decorated samples. This plasma-modified graphene could find use in, e.g., gas or liquid separation, or molecular sensing.

Graphene is a two dimensional material consisting in a monolayer of carbon atoms arranged in a honeycomb lattice. The combination of unique electrical, mechanical, thermal and optical properties in this one-atom thick material has made graphene the object of intense research activity in recent years [1]. Unfortunately, bringing this material into real-life applications on a large scale is far from straightforward. For instance, large-area pristine graphene is a semimetal with zero bandgap, which constitutes an obstacle for the development of graphene-based logic devices as gate modulation is not enough to produce definite off states [2]. Nevertheless, the bandgap as well as many other characteristics of graphene can be engineered by the introduction of several types of defects. Thus, significant attention has been very recently focused on the topic of defect generation in graphene, which has been accomplished by electron [3] *Corresponding author: Tel: (+34) 9851190 90. E-mail: rozada@incar.csic.es 
or ion irradiation [4], or alternatively by methods involving reaction with oxygen, such as heat treatment in an oxygen gas flow [5], UV-generated ozone oxidation [6] or oxygen plasma treatment [7-9]. Oxygen plasma treatment is an attractive method from a practical point of view, as it can be easily integrated in current circuit mass-production schemes, usually requires very short exposure times and the density of defects could be potentially controlled through the plasma processing parameters. Furthermore, unlike methods involving electron or ion irradiation that lead to defect populations of polydisperse type [3], certain oxygen plasmas enable the introduction of just one type of defect (e.g., atomic vacancies) [10]. The generation of a defect population of monodisperse type would be considerably advantageous, as it could provide a better control of the material properties. Of particular interest is the generation of atomic vacancies in graphene and especially multiatomic vacancies, because this type of defect is stable up to very high temperatures [11] and would thus be able to endure the temperatures required both during the fabrication process and at the operating conditions of the devices. Furthermore, most of the work on defect generation in graphene has been limited to micromechanically exfoliated samples, whereas little attention has been paid to graphene grown by chemical vapour deposition (CVD), which is much more application-wise.

Here, we report the use of microwave (MW)-generated oxygen plasma to introduce atomic-sized defects in CVD graphene with tunable densities from $\sim 10^{3}$ to $10^{5} \mu \mathrm{m}^{-2}$. The introduction of controlled densities of defects has been demonstrated and visualized down to the atomic scale by scanning tunneling microscopy (STM). The STM images reveal that these defects essentially comprise multiatomic vacancies of similar size and exhibit a regular distribution throughout the sample. To the best of our knowledge, this is the first time that such STM studies are performed for atomic vacancy-decorated graphene. In addition to the direct visualization of the defects and their density through STM, the introduction of structural disorder on the graphene samples has been confirmed independently by Raman spectroscopy. Finally, we investigate the effect of the atomic vacancies on the electronic transport properties of graphene by fabricating back-gated field-effect transistors and measuring their transfer characteristics (see experimental details in Supplementary information). We must stress that unlike other reports that investigated the electrical properties of graphene samples whose structure 
had been extensively damaged, here we asses this property for just slightly damaged graphene samples.

The generation of atomic-sized defects in graphene was accomplished by means of MW oxygen plasma, using appropriate plasma powers and exposure times to modulate the defect density. As this type of plasma proceeds mostly via chemical reactions, but not through ion bombardment, as is the case of, e.g., radio frequency oxygen plasma, the modification of carbon surfaces is more controllable. In fact, the introduction of atomic vacancies by microwave oxygen plasma using short exposure times has been previously demonstrated with HOPG [10]. Due to the short exposure times employed in our plasma treatments, the introduced defects consist exclusively of atomic vacancies comprising a few missing carbon atoms. These vacancies are generated via abstraction of carbon atoms by active oxygen species (for example, atomic oxygen), which give rise to $\mathrm{CO}$ and $\mathrm{CO}_{2}$ [12]. For short reaction times (i.e. short exposure times), only a few or several carbon atoms are abstracted at a given location on the graphite/graphene surface. However, if the reaction is allowed to continue for longer times, more and more carbon atoms from the vacancies will be abstracted, so that the initially small vacancies will grow laterally to encompass tens, hundreds or even thousands of missing carbon atoms, which correspond to pits of several to several tens of nanometers in diameter. Although pits in graphite with sizes above $1 \mathrm{~nm}$ are visualized by STM as pits or holes, smaller pits (i.e., atomic vacancies) are actually visualized by STM as apparent protrusions [12]. This situation is due to electronic effects, which are well known to have a prominent role in the formation mechanism of STM images. More specifically, in the case of atomic vacancies in graphite/graphene, the atoms surrounding these defects undergo an enhancement of the local density of electronic states close to the Fermi level and, because this partial electronic density is what STM actually probes, the vacancies are consequently seen as protrusions (bright spots) in the STM images. On the other hand, higher MW plasma powers generally led to larger numbers of defects, most probably because higher densities of species are available to react with the surface carbon atoms. As shown in the following, this method allowed tuning the defect density in CVD graphene roughly between $10^{3}$ and $10^{5} \mu \mathrm{m}^{-2}$.

Nanometer- and atomic-scale STM images of the graphene samples prior to [Fig 1(a)] and after [Fig. 1(b-f)] different oxygen plasma treatments are presented in this figure. The starting, pristine graphene exhibited a smooth but not atomically flat 
topography on the nanometer scale [height variations $<0.3 \mathrm{~nm}$; see Fig. 1(a)] that is assumed to reflect the topographical conformation of the $\mathrm{Cu}(111)$ supporting substrate. On the atomic scale [inset to Fig. 1(a)], the sample displayed the well-known honeycomb pattern that is expected for single-layer graphene when it is electronically decoupled from the supporting substrate. This fact, together with the absence of any other modulations in the atomic-scale STM images suggests a weak graphene- $\mathrm{Cu}(111)$ interaction. Images (b-e) of Fig. 1 correspond to samples treated with the MW oxygen plasma at $10 \mathrm{~W} / 9 \mathrm{~s}$ (b), $60 \mathrm{~W} / 3 \mathrm{~s}$ (c), $300 \mathrm{~W} / 1.5 \mathrm{~s}$ (d) and $390 \mathrm{~W} / 1.5 \mathrm{~s}(\mathrm{e})$. In contrast to their pristine graphene counterparts, the STM images of the plasma-treated samples revealed a number of apparent protrusions (bright spots) of more or less uniform appearance and regularly distributed throughout the graphene basal plane. An atomicscale STM image of one of these protrusions, corresponding to the $60 \mathrm{~W} / 3 \mathrm{~s}$ sample, is shown in Fig. 1(f).
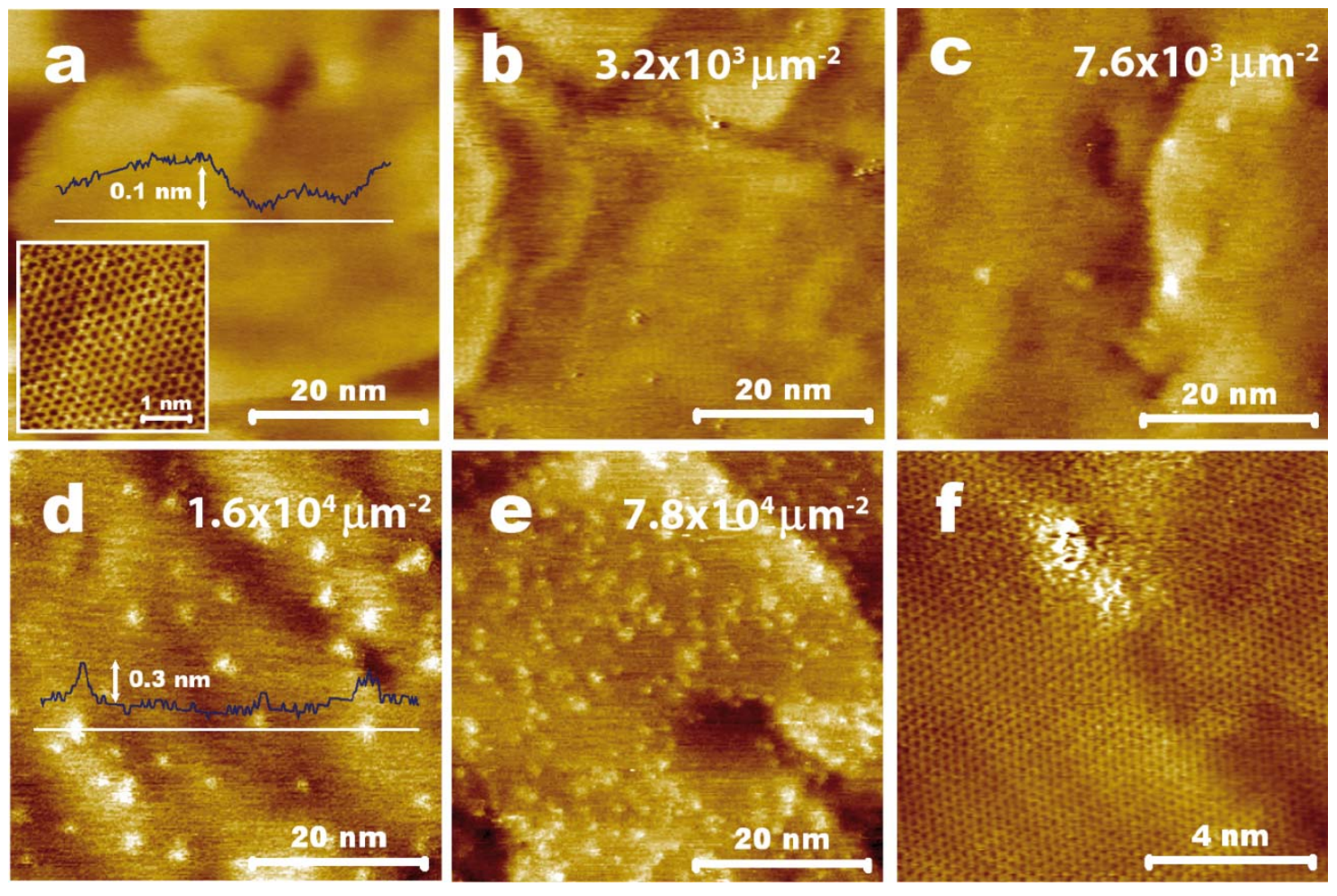

Figure 1: Nanometer-scale STM images for the pristine sample (a) and samples treated at $10 \mathrm{~W} / 9 \mathrm{~s}$ (b), $60 \mathrm{~W} / 3 \mathrm{~s}$ (c), $300 \mathrm{~W} / 1.5 \mathrm{~s}$ (d) and $390 \mathrm{~W} / 1.5 \mathrm{~s}$ (e). Atomic-scale STM images for the pristine sample (inset in a) and the sample treated at $60 \mathrm{~W} / 3 \mathrm{~s}$, showing one of the protrusions in more detail (f). Tunneling parameters: 200-500 pA (tunneling current) and 500-1000 mV (bias voltage) for nanometer-scale images and 600-700 pA (tunneling current) and $20 \mathrm{mV}$ (bias voltage) for atomic-scale images. 
According to previous work on graphite, the type of defect observed here by STM can be ascribed to atomic vacancies created through the removal of carbon atoms by atomic oxygen from the plasma [10]. The density of vacancies introduced by the plasma treatments could be readily determined from the STM images: $\sim 3.2 \times 10^{3} \mu^{-2}$ (b), $\sim 7.6 \times 10^{3} \mu \mathrm{m}^{-2}$ (c), $\sim 1.6 \times 10^{4} \mu \mathrm{m}^{-2}$ (d) and $\sim 7.8 \times 10^{4} \mu \mathrm{m}^{-2}$ (e). Due to the short exposure times used, in virtually all cases the observed protrusions exhibited similar heights $(\sim 0.2-0.4 \mathrm{~nm})$ and a narrow diameter distribution $(\sim 1-3 \mathrm{~nm})$ suggesting that all protrusions are associated with the same type of defect, i.e., small-sized multiatomic vacancies [10]. We note that larger vacancies, and even nanometer-sized holes, could be created for sufficiently long plasma exposure times. However, in that case small vacancies are also present because vacancy creation and enlargement take place continuously during the treatment, giving rise to polydisperse vacancy populations. By contrast, under proper conditions (i.e., short exposure times), this MW plasma-based method essentially afforded monodisperse populations of small multiatomic vacancies with controllable density, which could be useful for different applications of graphene, such as gas separation or water desalination [5]. It is also worth mentioning that, because this method is based on the use of reactive oxygen species, the introduction of oxygen functional groups attached to the vacancy edges is very likely to occur [10].

Raman spectroscopy is a non-destructive technique widely used in the investigation of graphene materials, in particular to provide structural information (e.g., presence of defects, stacking order, etc) [13]. Fig. 2(a) shows representative Raman spectra for the MW plasma-treated samples under investigation. The high crystalline quality of the starting, untreated graphene sample was confirmed by the complete absence of the defect-related D band at $\sim 1360 \mathrm{~cm}^{-1}$. After plasma treatment a noticeable D band appeared, and its intensity (in particular, the integrated intensity ratio of the D and G bands, $\mathrm{I}_{\mathrm{D}} / \mathrm{I}_{\mathrm{G}}$ ) was generally seen to increase in parallel with the density of atomic vacancies observed by STM. Therefore, the emergence of the D band can be directly related to the presence of this type of defect in the graphene lattice. On the other hand, the intensity of the 2D band (located at $\sim 2700 \mathrm{~cm}^{-1}$ ) underwent an obvious reduction after plasma treatment, which was also consistent with the development of structural disorder in the graphene lattice [14]. The dependence of the $\mathrm{I}_{\mathrm{D}} / \mathrm{I}_{\mathrm{G}}$ and $\mathrm{I}_{2 \mathrm{D}} / \mathrm{I}_{\mathrm{G}}$ ratios on the density of defects is quantitatively shown in Fig. 2(b) and (c), respectively. In addition to the evolution of the $\mathrm{D}$ and $2 \mathrm{D}$ bands, the $\mathrm{D}^{\prime}$ band emerged only for samples with 
defect densities larger than $\sim 10^{4} \mu \mathrm{m}^{-2}$. This band is seen as a shoulder of the $\mathrm{G}$ band at $\sim 1620 \mathrm{~cm}^{-1}$ and is also known to be induced by defects. Overall, the Raman data were consistent with the STM results in that they indicated the possibility to finely tune the density of defects (atomic vacancies) generated on graphene by MW oxygen plasma.
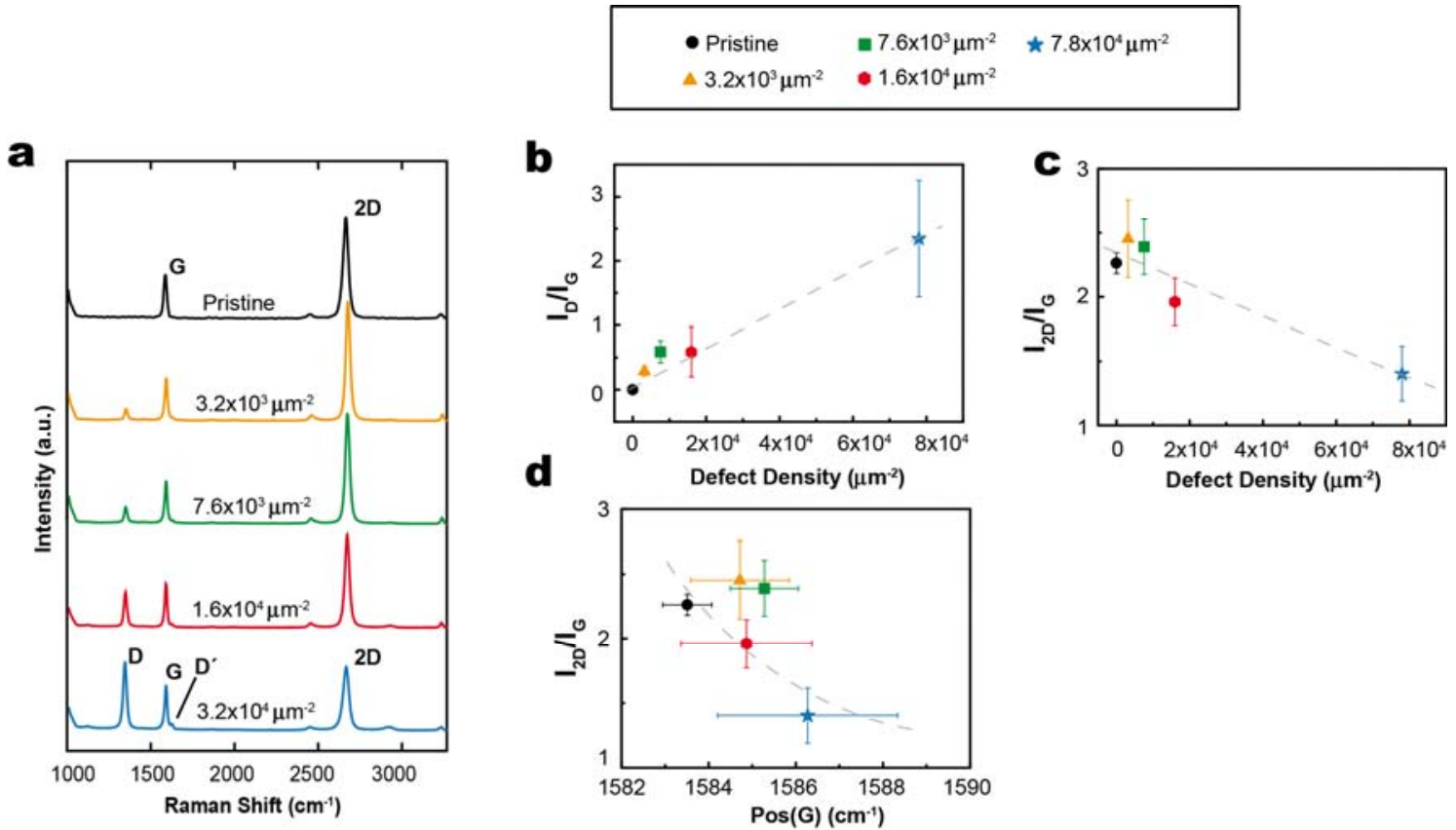

Figure 2: (a) Raman spectra for the pristine sample (black) and MW plasma-treated samples with defect densities $\sim 3.2 \times 10^{3} \mu \mathrm{m}^{-1}$ (orange), $\sim 7.6 \times 10^{3} \mu \mathrm{m}^{-1}$ (green), $\sim 1.6 \times 10^{4}$ $\mu \mathrm{m}^{-1}$ (red) and $\sim 7.8 \times 10^{4} \mu \mathrm{m}^{-1}$ (blue). All the spectra were measured after transferring on $\mathrm{SiO}_{2} / \mathrm{Si}$ substrates. $\mathrm{I}_{\mathrm{D}} / \mathrm{I}_{\mathrm{G}}$ (b) and $\mathrm{I}_{2 \mathrm{D}} / \mathrm{I}_{\mathrm{G}}$ (c) intensity ratios as a function of the density of defects. (d) $I_{2 D} / I_{G}$ intensity ratio versus the position of the $G$ band. Figures (b-d) show the average value of measurements at ten different spots, the error bars represent the standard deviation and the dashed lines are a guide to the eye.

The introduction of vacancy defects decorated with oxygen functionalities by the oxygen plasma can be expected to introduce some level of doping in the graphene sheets. Raman spectroscopy also provided some information about the electronic properties of defected graphene, such as the presence of doping [13]. In Fig. 2(d), the $I_{2 D} / I_{G}$ ratio has been plotted against the position of the $G$ band. For graphene samples that have been either $p$ - or $n$-doped, it has been reported that the $\mathrm{I}_{2 \mathrm{D}} / \mathrm{I}_{\mathrm{G}}$ ratio decreases and the position of the G band shifts to higher wavenumbers [13]. In our MW plasmatreated samples, the value of $\mathrm{I}_{2 \mathrm{D}} / \mathrm{I}_{\mathrm{G}}$ decreased from values over 2 for both pristine graphene and graphene decorated with a relatively low density of vacancies, to $\sim 1$ for 
the sample with the highest vacancy density. Concurrently, a shift of the G band from about 1583 to $1586 \mathrm{~cm}^{-1}$ was observed, suggesting chemical doping in the plasmatreated graphenes, although the actual doping type cannot be determined from these data.

Even though Raman spectroscopy is a powerful technique to identify doping in graphene, it also suffers from some degree of variability between samples that are produced under apparently identical conditions [15]. This effect is probably manifest in Fig. 2(d). More specifically, the pristine sample and the samples with defect densities of about $1.6 \times 10^{4}$ and $7.8 \times 10^{4} \mu \mathrm{m}^{-2}$ are highly consistent with each other. Nevertheless, there is some noticeable mismatch between these three samples and the samples with defect densities of about $3.2 \times 10^{3}$ and $7.6 \times 10^{3} \mu \mathrm{m}^{-2}$, although they are still broadly consistent with the general trends described above. Interestingly, these two sets of samples belong to different batches of the CVD graphene growth and transfer process. Hence, we tentatively attribute such differences to slight deviations in the fabrication process that are very difficult to control.

The electronic performance of the MW plasma-treated graphene samples was directly evaluated in back-gated field-effect transistors. Fig. 3(a) shows the gate voltage $\left(V_{G}\right)$ dependence of the drain current $\left(\mathrm{I}_{\mathrm{D}}\right)$ for the starting, pristine graphene as well as for plasma-treated samples with defect densities of about $1.6 \times 10^{4}$ and $7.8 \times 10^{4} \mu \mathrm{m}^{-2}$. For pristine graphene, we note that the charge neutrality point $\left(\mathrm{V}_{\mathrm{CNP}}\right)$ lies at $\sim 19 \mathrm{~V}$. Besides, instead of displaying perfect ambipolar behavior, the curve is slightly asymmetrical for holes and electrons. This phenomenon has been previously attributed to unintentional chemical doping that results from the microfabrication techniques and to the difference in work function between the graphene sample and its metal contacts $[15,16]$. One of the most important features observed in Fig. 3(a) is the upshift of the charge neutrality point for the defect-decorated samples compared to pristine graphene. Such upshift can be regarded as evidence of an enhancement in the p-type character of the plasma-treated samples [2]. In Fig. 3(b) it can be observed that the doping level correlates well with the density of atomic vacancies generated by the MW plasma. We note that each point in Figures 3 (b-e) represents the average value of measurements performed on ten different devices in order to make these results statistically meaningful. In addition to the upshift of the charge neutrality point, we see from Fig. 3(a) that the slope of the transfer curves 
on both sides of the charge neutrality point decreases in absolute value for plasmatreated graphene. The slope for gate voltages below (above) the charge neutrality point is directly related with the hole (electron) mobility. The mobility, $\mu$, of the charge carriers was computed through the formula $\mu=\left[\left(\partial I_{d} / \partial V_{g}\right) L\right] /\left[W C_{G} V_{d}\right]$ where $L$ and $W$ are respectively the length and width of the transistor channel, $V_{d}$ is the drain voltage and $C_{G}$ is the gate capacitance [2]. For a $\mathrm{SiO}_{2}$ substrate with a thickness of $300 \mathrm{~nm}, C_{G}$ $=1.15 \times 10^{-4} \mathrm{~F} \mathrm{~m}^{-2}$. Fig. 3(c) and (d) summarize the mobility values for holes and electrons. It is clear from these plots, as well as from the inset to Fig. 3(a), that the electron mobility degrades to a higher extent than the hole mobility upon introduction of atomic vacancies with the MW oxygen plasma. Such evolution towards unipolar behavior is consistent with a progressive p-type doping of the plasma-treated graphene samples.
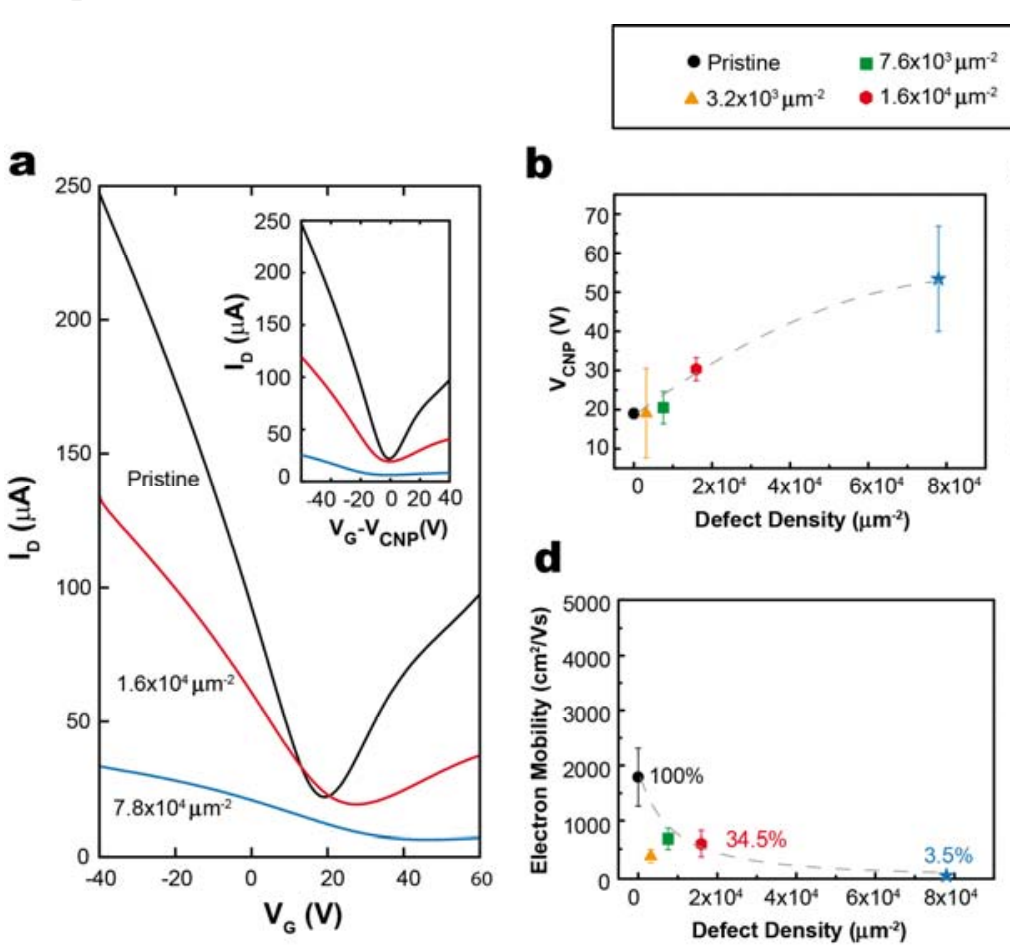

$\star 7.8 \times 10^{4} \mu \mathrm{m}^{2}$

C

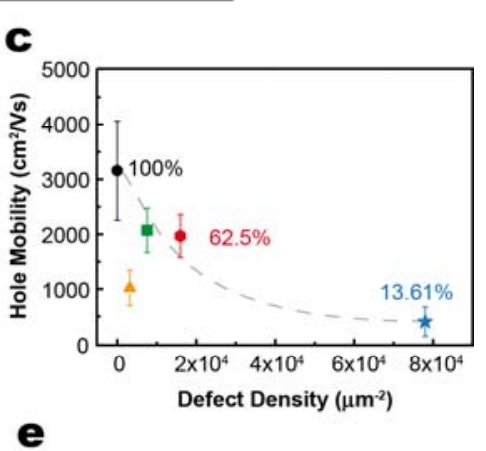

e

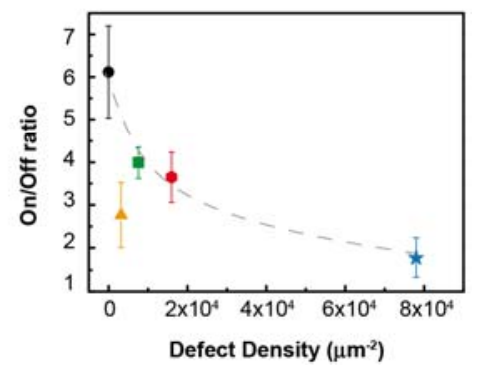

Figure 3: (a) $V_{G}$ dependence of $I_{D}$ for the pristine sample (black plot) and MW plasmatreated samples with defect densities $\sim 1.6 \times 10^{4} \mu \mathrm{m}^{-1}$ (red) and $\sim 7.8 \times 10^{4} \mu \mathrm{m}^{-1}$ (blue). The inset in (a) plots $I_{D}$ against $V_{G}-V_{C N P}$, showing the dopant-induced asymmetry for holes $\left(\mathrm{V}_{\mathrm{G}}-\mathrm{V}_{\mathrm{CNP}}<0\right)$ and electrons $\left(\mathrm{V}_{\mathrm{G}}-\mathrm{V}_{\mathrm{CNP}}>0\right)$. The charge neutrality point position (b), the mobility of holes (c) and electrons (d), and the on/off ratio (e) are plotted as a function of the density of defects. Figures (b-e) show the average value of measurements performed on ten different devices, the error bars represent the standard deviation and the dashed lines are a guide to the eye. 
In principle, the reduction in the charge carrier mobility can be ascribed either to scattering of the charge carriers at the defect sites that have been generated by the plasma treatment or to the opening of a bandgap [2]. The opening of a bandgap in graphene heavily decorated with oxygen groups (O/C atomic ratio of 0.5$)$ has been theoretically predicted under the assumption that the graphene lattice retains its structural integrity [7]. However, this premise is not fulfilled in the present case, because the plasma treatment causes the subtraction of carbon atoms from the graphene lattice, thus resulting in a significant modification of the electronic properties (e.g., decrease in the charge carrier mobility). Moreover, even if the model could be applied to our samples, the amount of oxygen introduced in the present case would be insufficient by itself to significantly change the band structure of graphene (atomic scale STM images indicate that the presence of oxygen groups must be restricted to vacancy edges, yielding estimated $\mathrm{O} / \mathrm{C}$ atomic ratios of the order of 0.01 ). More significantly, an increase in the on/off ratio of the FET would be expected if a bandgap was opened in graphene, whereas in our case the measured on/off ratio tended to decrease for all the plasma-treated samples [Fig. 3(e)]. On the other hand, point defects in the graphene network are known to act as scattering centers for the charge carriers [17], thus inducing a lowering in the mobility as that observed in our case. In the case that a bandgap is not opened, this reduced mobility would also induce a decrease of the on/off ratio measured on FET devices. Therefore, we conclude that the introduction of isolated vacancies in graphene is responsible for the observed decreased mobility in our samples, but at the defect densities attained in this work a gap is not opened in the electronic band structure of graphene.

In summary, we have presented a MW oxygen plasma-based method for the introduction of small, similar-sized atomic vacancies in graphene with controlled density, in the range from about $10^{3}$ to $10^{5} \mu^{-2}$. The electronic properties of graphene undergo major changes as a result of its decoration with this type of defect. For example, we observed degradation in the mobility of both electrons and holes, although a net excess of positive charge carriers (p-type doping) was measured, the latter of which can be tentatively accounted for by the saturation of the vacancy edges with oxygen functional groups. We envisage that this method can be used for the tailored generation of tiny holes in graphene, which could then be used as membranes for, e.g., gas or liquid separation, DNA sequencing or molecular sensing. Furthermore, several 
schemes aimed at imparting specific chemical functionalities to graphene involve the introduction of atomic vacancies, so the present method can be used as a convenient starting point towards this end.

\section{Acknowledgements}

Financial support from the Spanish MINECO and the European Regional Development Fund (project MAT2011-26399) is gratefully acknowledged. Part of this work was supported by PRESTO, Japan Science and Technology. P.S.-F- acknowledges the receipt of a postdoctoral fellowship from JSPS. R. R. is grateful for the receipt of a predoctoral contract (FPU) from the Spanish MECD.

\section{References}

[1]Novoselov KS, Fal V, Colombo L, Gellert P, Schwab M, Kim K. A roadmap for graphene. Nature 2012;490(7419):192-200.

[2]Schwierz F. Graphene transistors. Nat Nanotechnol 2010;5(7):487-96.

[3]Robertson AW, Allen CS, Wu YA, He K, Olivier J, Neethling J, et al. Spatial control of defect creation in graphene at the nanoscale. Nat Commun 2012;3:1144.

[4] Kim J-H, Hwang JH, Suh J, Tongay S, Kwon S, Hwang CC, et al. Work function engineering of single layer graphene by irradiation-induced defects. Appl Phys Lett 2013;103(17):171604.

[5]Yamada Y, Murota K, Fujita R, Kim J, Watanabe A, Nakamura M, et al. Subnanometer vacancy defects introduced on graphene by oxygen gas. J Am Chem Soc 2014;136(6):2232-5. [6]Yang D-P, Wang X, Guo X, Zhi X, Wang $\mathrm{K}$, Li C, et al. UV/O $\mathrm{O}_{3}$ generated graphene nanomesh: formation mechanism, properties, and FET studies. J Phys Chem C 2014;118(1):725-31. [7]Nourbakhsh A, Cantoro M, Vosch T, Pourtois G, Clemente F, van der Veen MH, et al. Bandgap opening in oxygen plasma-treated graphene. Nanotechnology 2010;21(43):435203. [8]Xie G, Yang R, Chen P, Zhang J, Tian X, Wu S, et al. A general route towards defect and pore engineering in graphene. Small 2014;10(11):2280-4.

[9]Mao H, Wang R, Zhong J, Zhong S, Chen W. Mildly $\mathrm{O}_{2}$ plasma treated CVD graphene as a promising platform for molecular sensing. Carbon 2014;76:212-9.

[10]Paredes JI, Solís-Fernández P, Martínez-Alonso A, Tascón JMD. Atomic vacancy engineering of graphitic surfaces: controlling the generation and harnessing the migration of the single vacancy. J Phys Chem C 2009;113(23):10249-55.

[11]Banhart F, Kotakoski J, Krasheninnikov AV. Structural defects in graphene. ACS Nano 2011;5(1):26-41.

[12]Paredes JI, Martínez-Alonso A, Tascón JMD. Early stages of plasma oxidation of graphite: nanoscale physicochemical changes as detected by scanning probe microscopies. Langmuir 2002;18(11):4314-23.

[13]Ferrari AC, Basko DM. Raman spectroscopy as a versatile tool for studying the properties of graphene. Nat Nanotechnol 2013;8(4):235-46.

[14]Rozada R, Paredes JI, Villar-Rodil S, Martínez-Alonso A, Tascón JMD. Towards full repair of defects in reduced graphene oxide films by two-step graphitization. Nano Res 2013;6(3):21633. 
[15]Casiraghi C, Pisana S, Novoselov KS, Geim AK, Ferrari AC. Raman fingerprint of charged impurities in graphene. Appl Phys Lett 2007;91(23):233108.

[16]Orofeo CM, Hibino H, Kawahara K, Ogawa Y, Tsuji M, Ikeda K-i, et al. Influence of Cu metal on the domain structure and carrier mobility in single-layer graphene. Carbon

2012;50(6):2189-96.

[17]Chen JH, Cullen WG, Jang C, Fuhrer MS, Williams ED. Defect scattering in graphene. Phys Rev Lett 2009;102(23):236805. 


\title{
Controlled generation of atomic vacancies in chemical vapor deposited graphene by microwave oxygen plasma
}

\author{
R. Rozada ${ }^{\text {a* }}$, P. Solís-Fernández ${ }^{\mathrm{b}}$, J.I. Paredes ${ }^{\mathrm{a}}$, A. Martínez-Alonso $^{\mathrm{a}}$, H. Ago ${ }^{\mathrm{b}}$, J.M.D. \\ Tascón ${ }^{\mathrm{a}}$ \\ anstituto Nacional del Carbón, INCAR-CSIC, Apartado 73, 33080 Oviedo, Spain \\ ${ }^{b}$ Institute for Materials Chemistry and Engineering, Kyushu University, Fukuoka 816- \\ 8580, Japan
}

\section{Supplementary Information}

\section{Experimental}

Single-layer CVD graphene was grown at ambient pressure over an heteroepitaxial thin $\mathrm{Cu}(111)$ film deposited on c-plane sapphire, following a method described elsewhere [1]. The CVD parameters were adjusted to obtain a complete coverage of high quality graphene. Briefly, a pre-annealing was conducted at $1000{ }^{\circ} \mathrm{C}$ for 40 minutes in an $\mathrm{Ar} / \mathrm{H}_{2}$ gas flow $\left(\mathrm{H}_{2}\right.$ concentration $2.5 \%$ ), and then the temperature was raised to $1075{ }^{\circ} \mathrm{C}$ and $\mathrm{CH}_{4}$ in a concentration of $10 \mathrm{ppm}$ was added to the gas flow for 1 hour. The controlled generation of atomic vacancies on the as-grown graphene samples was performed in a MW-induced oxygen plasma apparatus (Technics Plasma 200-G system). $\mathrm{O}_{2}(99.999 \%$ pure) was used for the treatment and the working pressure was set to 1.0 mbar. Proper combinations of plasma power and exposure time allowed tuning the density of defects created on graphene. Specifically, the selection of particular plasma power values and exposure times was the result of a feedback process that included visualizing by STM the appearance and density of defects generated in preliminary experiments. We have performed control experiments to check for the reproducibility of the results by preparing several plasma-etched specimens using the same processing conditions. We observed that identical treatments yielded essentially the same results and that the graphene samples, which were about $1 \times 1 \mathrm{~cm}^{2}$ in size, became decorated with essentially the same density and type of defects everywhere as deduced by examining many different regions with STM. STM imaging was performed with a Nanoscope IIIa Multimode apparatus (Veeco Instruments) under ambient conditions using mechanically *Corresponding author: Tel: (+34) 9851190 90. E-mail: rozada@incar.csic.es 
prepared $\mathrm{Pt} / \mathrm{Ir}(80 / 20)$ tips. Images were recorded in the constant current mode with typical tunneling parameters of $200-500 \mathrm{pA}$ and $500-1000 \mathrm{mV}$ for nanometer-sized images and of 600-700 pA and $20 \mathrm{mV}$ in the case of atomic-scale images. Following STM characterization, the graphene samples were transferred to a $\mathrm{SiO}_{2}(300 \mathrm{~nm}) / \mathrm{Si}$ substrate by first coating them with poly(methyl methacrylate) (PMMA) and etching the $\mathrm{Cu}$ film in a $\mathrm{FeCl}_{3}$ solution. After transferring the PMMA/graphene film to the $\mathrm{SiO}_{2} / \mathrm{Si}$ substrate, the polymer was dissolved using hot acetone. Raman characterization of the graphene samples on $\mathrm{SiO}_{2} / \mathrm{Si}$ was carried out at ambient conditions with a Nanofinder 30 confocal Raman microscope (Tokyo Instruments Inc.) using a laser excitation wavelength of $532 \mathrm{~nm}$. The spot size was about $600 \mathrm{~nm}$ and a low incident laser power $(\sim 1.6 \mathrm{~mW})$ was used to avoid damage to the samples. Peaks in Raman spectra were fitted to Lorentzian functions. For the electronic transport measurements, $10 \mu \mathrm{m}$-wide lines of graphene were patterned by photolithography, combined with oxygen plasma to remove the rest of the graphene film. Then, electrodes were made by photolithography followed by thermal evaporation of $\mathrm{Au}$. The transport properties were measured under vacuum $\left(\sim 5 \times 10^{-4} \mathrm{~Pa}\right)$ at room temperature using an Agilent B1500A (Agilent Technologies) semiconductor device analyzer.

\section{References}

[1]Hu B, Ago H, Ito Y, Kawahara K, Tsuji M, Magome E, et al. Epitaxial growth of large-area single-layer graphene over $\mathrm{Cu}(111) /$ sapphire by atmospheric pressure CVD. Carbon 2012;50(1):57-65. 